\title{
Desigualdades intraurbanas em internações hospitalares por doenças respiratórias e circulatórias em uma área da cidade de São Paulo
}

\author{
Intra-urban inequalities in hospitalizations for respiratory \\ and circulatory diseases in an area of the city of São Paulo
}

Helena Ribeiro

Edelci Nunes Silva

\section{Resumo}

As dinâmicas existentes em áreas urbanas apresentam consequências à saúde, relacionadas aos determinantes sociais e ambientais, à promoção da saúde e à atenção primária. 0 artigo mapeia e analisa as internações hospitalares por doenças respiratórias e circulatórias de idosos e respiratórias em crianças por distritos em área da cidade de São Paulo, comparando-as com a distribuição espacial do índice de desenvolvimento, da qualidade socioambiental, da presença de favelas, a partir de dados secundários públicos e do georreferenciamento com o programa ArcGis 9.2. As internações por doenças do aparelho circulatório e respiratório em idosos apresentaram padrão socioespacial. Maiores taxas estão relacionadas aos distritos com pior perfil socioambiental e baixo IDH e taxas menores estão associadas aos distritos com melhor perfil socioambiental e alto IDH. Não houve um padrão socioespacial definido das internações de crianças. A espacialização da morbidade refletiu as desigualdades na cidade de São Paulo.

Palavras-chave: saúde urbana; desigualdade; internação hospitalar; doença respiratória; doença circulatória.

\begin{abstract}
The dynamics that exist in urban areas present consequences to health, related to social and environmental determinants, health promotion and primary care. The article maps and analyzes hospitalizations for respiratory and circulatory diseases in elderly individuals and for respiratory diseases in children by district in an area of the city of São Paulo. It compares them to the spatial distribution of the human development index, of socio-environmental quality, and of the presence of slums, using public secondary data and georeferencing through the software ArcGis 9.2. Hospitalizations due to circulatory and respiratory diseases among the elderly showed a sociospatial pattern. Higher rates occur in the districts with worse socio-environmental profile and low $\mathrm{HDI}$, and lower rates occur in districts with better socio-environmental profile and high HDI. No socio-spatial pattern was found for children's hospitalizations. The space distribution of morbidity reflected inequalities in the city of São Paulo.
\end{abstract}

Keywords: urban health; inequalities; hospitalization; respiratory disease; circulatory disease. 


\section{Introdução}

A cidade de São Paulo, com aproximadamente 12 milhões de habitantes (IBGE, 2015) e uma área de $1.521 \mathrm{~km}^{2}$, incorporou as novas demandas do modelo econômico global e reafirmou seu status de metrópole global. É o centro de comando, de negócios e de fluxos de informações, que concentra atividades de serviços e constitui um importante polo econômico, no País e no mundo.

Até a década de 1970, a organização do espaço da cidade baseou-se no padrão centro-periferia, ou seja, as áreas centrais e dotadas de infraestrutura urbana foram destinadas à população de mais alta renda, enquanto as áreas periféricas, distantes do centro, e carentes de infraestrutura foram ocupadas pelas camadas mais pobres da população.

Nas décadas seguintes, as transformações foram impulsionadas pela globalização contemporânea, "um fenômeno multifacetado com dimensões econômicas, sociais, políticas, culturais, religiosas e jurídicas interligadas de modo complexo" (Souza Santos, 2002). 0 aumento de abertura das fronteiras ao comércio e aos fluxos de capital econômico, a crescente incorporação tecnológica, a ampliação dos meios de comunicação a novas tecnologias digitais, a migração de populações, em busca de melhores condições de vida e trabalho (Ribeiro e Vargas, 2015), provocaram a modificação do padrão centro-periferia. As novas necessidades do mercado e do capital levaram à incorporação e a investimentos em novas áreas e à desvalorização de algumas regiões centrais, já consolidadas. Esse modelo se caracterizou pelo aprofundamento do processo de segregação intraurbana e pela polarização da vida social.
"O município de São Paulo, impulsionado pelo desenvolvimento econômico e pelo amplo fluxo migratório, teve como consequência concentrar pessoas, riquezas, mas também desigualdades" (Peres e Ruotti, 2015).

A cidade tem, portanto, nos seus interstícios, um mosaico complexo de espaços ricos e pobres, altamente segregados. Há presença de bairros nobres lado a lado com favelas; espaços verticalizados e consolidados com presença de cortiços e de sem-teto. As favelas crescem na periferia, mas também estão presentes nos setores ricos, recém-incorporados pelo capital (Taschner, 2001; Santos e Silveira, 2001e Almeida, 2001). Como em outras regiões metropolitanas do País, "crescem as áreas habitadas por pessoas em moradias precárias, formando ocupações irregulares que afetam as condições de saúde da população" (Aith e Scalco, 2015).

Há, concomitantemente, uma tendência de modificação do perfil populacional. A população da cidade vem envelhecendo. 0 percentual da população acima de 60 anos aumentou, entre 1970 e 1991 , de $6,08 \%$ para $8,08 \%$ (Taschner, 2001, p. 35): em 2007, representava $11 \%$ da população total (IBGE, 2007) e, em 2015, a população de idosos correspondia a 13,5\% (Seade, 2016). Na periferia, o percentual também vem aumentando. Nas décadas anteriores a 1991 era 3\%; em 1991, 4,60\%; e, em $1996,4,99 \%$ da população tinha 60 ou mais anos (Taschner, 2001, p. 35).

Essa dinâmica espacial e populacional se, por um lado, cria uma cidade heterogênea, por outro, concentra, em algumas áreas "nobres", dotadas de serviços e infraestrutura, a população afluente e áreas com população de baixa renda, com todo tipo de carência - de moradia, de emprego, serviços, infraestrutura básica, 
como água, esgoto, etc. e de saúde, educação, cultura, lazer - aprofundando os problemas sociais e mantendo a população na espiral da pobreza (Santos, 1996, p. 10)

Uma das dimensões da degradação das condições de existência são as condições socioambientais e de saúde a que estão submetidas parcelas da população, revelando mais uma dimensão da pobreza e da iniquidade urbanas.

Ribeiro (2006) e Santos (2003) ressaltam, porém, que a urbanização em si não é um mal. Os autores argumentam que, do ponto de vista da saúde, a urbanização trouxe muitos benefícios, tanto no nível individual, quanto no coletivo. De modo geral, houve queda nas taxas de mortalidade, mortalidade infantil tardia e aumento na expectativa de vida, em função dos vários benefícios trazidos pelos equipamentos urbanos, como o acesso a água potável, saneamento básico, energia, bem como pelos programas de vacinação, de complementação alimentar, acesso aos serviços médicos, educação, informação, etc.

0 provimento de serviços de saneamento e de saúde pública, ainda que de forma não universalizada, levou a uma mudança no perfil da morbimortalidade. Nas cidades, o surgimento e 0 agravamento das patologias estão muito ligados ao modo de vida, às desigualdades sociais e ambientais (Ribeiro, 2006; Jara et al., 2010).

No meio urbano paulistano, "as patologias mais relevantes estão relacionadas às faixas etárias, ao ambiente social e aos impactos ambientais das diversas poluições" (Ribeiro, 2006, p. 292).

A apreensão dos impactos do ambiente na saúde deve, portanto, considerar esse complexo processo de transformações: espaciais, populacionais, sociais e ambientais, ocorrido nas áreas urbanas e, mais especificamente, nas cidades dos países em desenvolvimento, nas últimas décadas do século XX e início do século XXI (Opas, 2011).

As dinâmicas existentes, em áreas urbanas, apresentam consequências à saúde, relacionadas aos determinantes sociais e ambientais, à promoção da saúde e à atenção primária. 0 crescimento urbano não planejado e insustentável exerce pressão sobre os serviços básicos e dificulta 0 atendimento de necessidades de uma população diversa, fazendo com que as disparidades sociais aumentem no interior das cidades, com repercussões importantes na saúde (ibid.). Em 2011, a Organização Pan-americana de Saúde aprovou Estratégia e Plano de Ação sobre a Saúde Urbana para atender às necessidades sanitárias específicas da população urbana das Américas, com base em 5 princípios orientadores: equidade, sustentabilidade, desenvolvimento sustentável, segurança humana e bom governo.

Para atender ao princípio da equidade, é necessário que se conheça a situação de saúde da população urbana e de sua relação com o espaço social e ambiental.

Apesar de os registros das informações em saúde, no Brasil, disponibilizarem os bancos de dados de mortalidade e internações hospitalares na rede mundial de computadores, permitindo verificar sua distribuição espacial, poucos estudos têm sido feitos analisando a distribuição espacial intraurbana, para identificar e entender as desigualdades em saúde no interior das cidades. Barata (2012) ressalta a importância da análise dos eventos de saúde, em relação ao local de moradia, tomando o espaço geográfico como indicativo 
das condições de vida da população que nela reside, para abordar as desigualdades sociais no plano de agregados.

Esses dados, contudo, apresentam limitações. Por exemplo, os dados de internação são tratados para efeitos de pagamento e repasse de recursos financeiros do Sistema Único de Saúde aos hospitais, e não com fins epidemiológicos; e os dados de mortalidade estão disponíveis, somente, na escala temporal mensal. Mesmo assim, é possível realizar estudos com essas informações, que indicam diferenças espaciais, e correlacioná-los com outras variáveis. A saúde depende de características individuais, físicas e psicológicas e, também, do ambiente mais próximo das pessoas, do ambiente macrorregional e do global (Aith e Scalco, 2015).

Os registros existentes e disponíveis referem-se à ponta do iceberg das condições de adoecimento, de modo que pequenos incômodos e problemas de saúde, que não levam à internação hospitalar, requerem a realização de inquéritos de saúde, os quais podem ser muito custosos e demorados.

Sendo assim, a cartografia, e, principalmente, os Sistemas de Informação Geográfica SIG/GIS, consiste em ferramenta importante para os pesquisadores, pois, através dela, é possível localizar o evento no espaço e relacioná-lo às variáveis socioambientais, a fim de lançar hipóteses sobre o porquê de sua ocorrência ou de obter explicações relacionadas aos fatores espaciais, permitindo melhorar 0 conhecimento dos processos de saúde e de doença. Adicionalmente, os SIGs permitem monitorar as desigualdades em saúde, ao fazer simultaneamente, uma análise múltipla de vários determinantes de saúde, em diferentes níveis de agregação. Um dos primeiros passos para se determinar a magnitude das iniquidades em saúde é a medição das desigualdades. Elas podem indicar as necessidades não satisfeitas de serviços de saúde, assim como populações vulneráveis (Loyola; Castillo-Salgado; Nájera-Aguilar et al., 2002).

Este trabalho tem como objetivo verificar a distribuição espacial de residentes internados em hospitais públicos, em uma área da cidade de São Paulo, Brasil, com mapeamento de dados de internação hospitalar por doenças respiratórias, em crianças; e respiratórias e circulatórias, em adultos maiores de 60 anos, utilizando a série temporal de 2003 a 2007. Essa série temporal foi utilizada porque os dados finalizados de internação hospitalar, por endereço de residência dos pacientes, demoram a ser disponibilizados pelo Datasus aos pesquisadores, por algumas razões: dependem da alta do paciente para que os dados consolidados sejam processados, e esta pode demorar, em alguns casos; dependem de verificação e correção do endereço de residência dos pacientes; só são liberados em casos especiais de pesquisa científica, em que os endereços individuais não sejam revelados, por questões éticas. Nesse caso, os dados foram obtidos para tese de doutorado, realizada na Faculdade de Saúde Pública da USP, e serão publicados após a defesa.

\section{Metodologia}

Para a realização do presente estudo, foram utilizados dados secundários de morbidade referentes às internações hospitalares registradas nas AlHs - Autorização de Internações Hospitalares - obtidos no Sistema de Informações Hospitalares do Sistema Único de Saúde 
(SIH/SUS) (Datasus, 2016), de pessoas residentes em 14 distritos do município de São Paulo: Cidade Ademar, Cidade Dutra, Campo Belo, Campo Grande, Cursino, Socorro, Itaim Bibi, Jabaquara, Moema, Pedreira, Sacomã, Santo Amaro, Saúde e Vila Mariana (Figura 1).

Esses distritos foram escolhidos por conterem, no seu território, duas estações meteorológicas representativas das características climáticas da cidade de São Paulo. 0 clima é um indicador ambiental importante na avaliação do desencadeamento das doenças estudadas e, por isso, os distritos escolhidos são representativos de condições climáticas semelhantes, mas de perfil sociodemográfico diverso do município de São Paulo. Ou seja, a área de estudo abrange distritos com excelentes a péssimas condições socioambientais e, por isso, foi selecionada para pesquisar desigualdade em saúde, tendo o clima como controle. Segundo Barata (2012, p. 35), "a vantagem de utilizar espaços geográficos como indicadores de condições de vida está em tomar a complexidade de organização social em seu todo".

Figura 1 - Localização dos distritos estudados no município de São Paulo
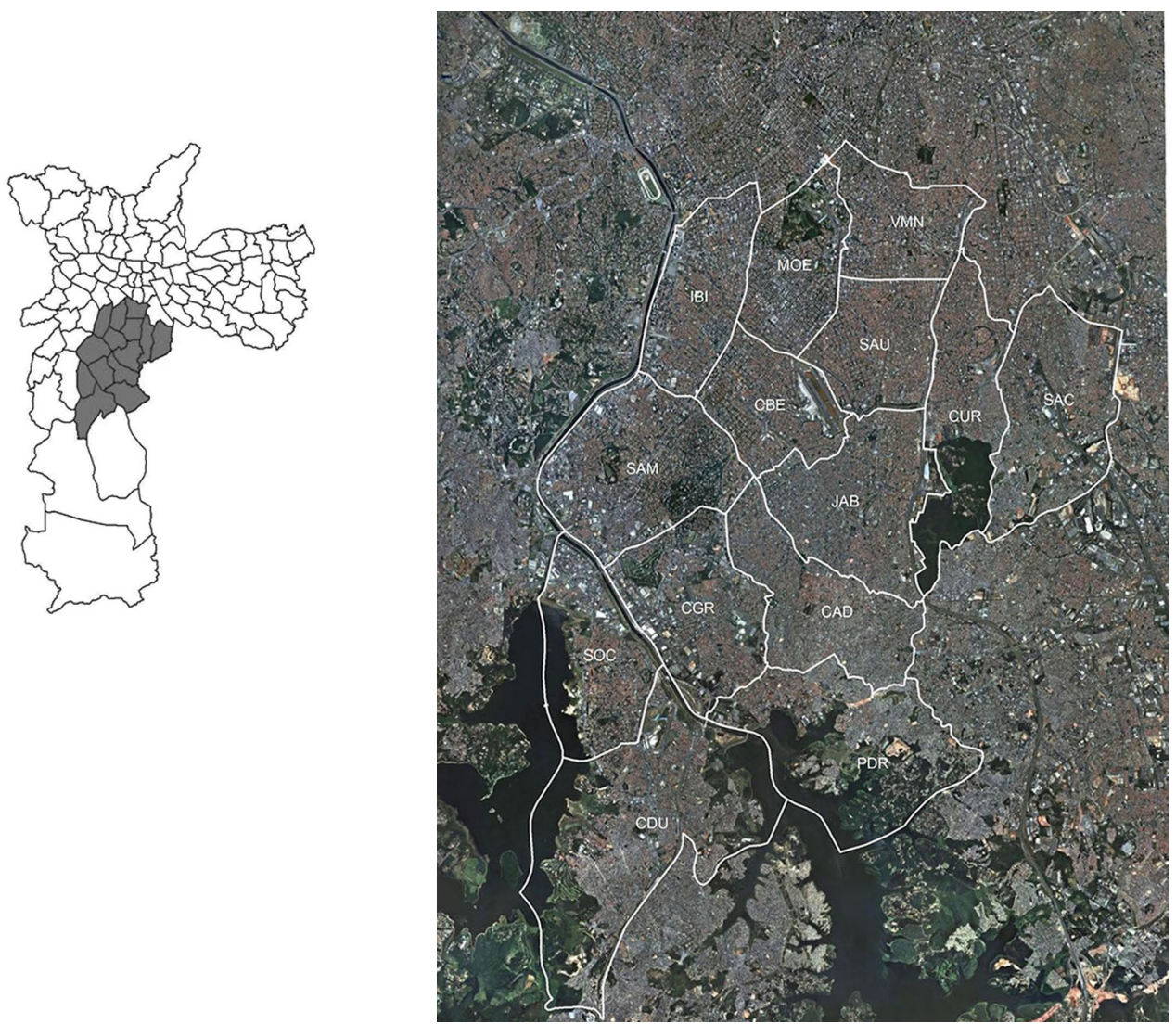
A fim de nortear as análises da distribuição espacial das internações hospitalares, informações sobre os aspectos dos distritos foram mapeadas: concentração de favelas, perfil socioambiental, índice de desenvolvimento humano - IDH e população.

A população estudada foi de crianças menores de 5 anos, para as internações por doenças respiratórias, e o grupo etário de 60 anos ou mais, para doenças do aparelho circulatório e respiratório. São os 2 grupos etários com maior vulnerabilidade aos impactos negativos do ambiente.

A partir do conjunto de dados do estado de São Paulo, foram selecionadas as informações relativas ao município de São Paulo código 355030 e, posteriormente, as doenças conforme a versão 10 da Classificação Internacional de Doenças - CID 10 - Capítulo 9 Doenças do Aparelho Circulatório (100 - 199) e Capítulo 10 - Doenças Respiratórias (J00-J32; J40-J47; J80-J99) de 2003 a 2007.

O SIH-SUS é um banco de dados administrativo, cujo objetivo é viabilizar o pagamento dos serviços prestados pelo Sistema Único de Saúde - SUS e que contém informações sobre as internações hospitalares. Assim, uma internação ocorrida em um determinado ano pode ser processada no ano seguinte à internação ou nos anos subsequentes. Por exemplo, no banco de dados de 2004, há informação de internações ocorridas em 2003, 2002, 2001, 2000, e assim por diante, ou seja, as informações referem-se ao ano em que os procedimentos com a internação foram pagos, e não ao ano em que ela ocorreu. Dessa forma, para obter o conjunto das informações de internação ocorridas em um determinado ano, os dados foram reunidos em uma planilha única (2003 a 2008).

0 georreferenciamento dos dados foi feito a partir do Código de Endereçamento Postal (CEP) de residência do paciente, utilizando o programa ArcGIS versão 9.2. Foi utilizada a base de ruas com projeção SAD69. Nesse procedimento, houve perda de informações devido à não localização dos CEPs. Os endereços cujos CEPs não foram localizados e as informações duplicadas foram excluídos da análise.

Apesar de ser possível a desagregação da informação até o nível do setor censitário, no presente trabalho, optou-se por utilizar a escala do distrito por duas razões: a) falta de informação sobre a projeção populacional no nível do setor censitário; e b) muitos dados de internação se concentraram no setor censitário dos hospitais.

As internações por doenças do apareIho circulatório - Capítulo 9 - correspondem a 24.318 casos, ou $8,6 \%$ do total de todas as internações no período de 2003 a 2007, no setor estudado. As doenças respiratórias correspondem a 12.269 casos de crianças menores de 5 anos, ou 5,0\%; e 8.894, ou 3,7\% do total de internações de pessoas acima de 60 anos, respectivamente.

As taxas anuais foram calculadas, por distrito, e padronizadas por idade, conforme fórmula abaixo:

$$
\mathrm{Tx} .=\frac{\text { número de internações/ano no distrito }}{\text { população da faixa etária no distrito }} \times 10.000 \mathrm{hab} \text {. }
$$

Os mapas temáticos foram feitos no software ArcGIS versão 9.2. 


\section{Características dos distritos}

A região selecionada é cortada por importantes vias e de tráfego intenso - como a Avenida Nações Unidas (marginal do Rio Pinheiros), a Avenida dos Bandeirantes, a Rodovia Imigrantes, que faz ligação com o Litoral de São Paulo, entre outras - e tem presença significativa de favelas.

Áreas de favelas estão em todos os distritos selecionados, exceto o distrito de Moema. Na Figura 2 é possível verificar a maior concentração, em número e em tamanho, de áreas de favelas nos distritos do Jabaquara, Cidade Ademar, Cidade Dutra, Pedreira e Sacomã.

$\mathrm{O}$ IDH apontou os distritos do Ibirapuera e de Moema com os melhores indicadores de desenvolvimento humano (alto nível). Por outro lado, os distritos de Cidade Ademar, Cidade Dutra e Pedreira apresentam os piores indicadores (nível baixo) (Figura 3).

Os distritos de Santo Amaro, Socorro, Campo Belo e Moema são aqueles que apresentam melhor qualidade socioambiental, situando-se nos primeiros grupos. Os distritos de Campo Grande, Itaim Bibi, Vila Mariana, Cursino, Saúde, Pedreira e Cidade Dutra são aqueles que apresentam características intermediárias, do ponto de vista socioambiental; enquanto os distritos de Sacomã, Jabaquara e Cidade Ademar são os que apresentam as piores condições socioambientais, segundo a classificação da Secretaria do Verde e Meio Ambiente do Município - SVMA (Sepe e Tokiya, 2004) (Figura 4).

Os distritos de Cidade Ademar, Cidade Dutra e Pedreira apresentam os piores indicadores de IDH e socioambiental, bem como concentram, em seu território, maiores quantidades de favela.

A Figura 5 mostra a proporção, por distrito, da população (\%) de crianças residentes, em relação ao total da população do setor estudado. A distribuição mostra que os distritos mais centrais apresentam menores taxas de população infantil (menos de $5 \%$ ), enquanto os distritos do Sacomã, Jabaquara, Cidade Dutra apresentam até $15 \%$ da população de crianças menores de 5 anos, e o distrito de Cidade Ademar apresenta a maior proporção de crianças menores de 5 anos, com percentual acima de $15 \%$ do total da população do setor.

A Figura 6 mostra que a população de mais de 60 anos está concentrada nos distritos de Vila Mariana, Sacomã e Jabaquara - mais de $10 \%$ da população do setor -, enquanto os distritos de Socorro e Pedreira contêm menos de $5 \%$ da população com mais de 60 anos. Os distritos restantes abrigam até $10 \%$ da população de mais de 60 anos em seus territórios.

Estudo sobre os usuários do Sistema Único de Saúde (SUS), no município de São Paulo, indicou que, aproximadamente, 50\% da população residente depende exclusivamente do atendimento do SUS, mas a distribuição é desigual. Nos distritos estudados, 7 têm porcentagem de usuários inferior a 40\%: Moema, Itaim Bibi, Campo Belo, Vila Mariana, Morumbi, Santo Amaro, Saúde. Os distritos com usuários acima de $40 \%$ são Cursino, Socorro, Sacomã, Jabaquara, Cidade, Dutra, Cidade Ademar, e somente o distrito de Pedreira tem mais de $60 \%$ da população residente usuária exclusivamente do Sistema Único de Saúde (CEInfo, 2010)

Trata-se, também, de uma região muito diversificada, em relação ao padrão de ocupação. Pode-se, em um mesmo distrito, 
Figura 2 - Distribuição das áreas de favelas, por perímetro (m), no setor, município de São Paulo, SP

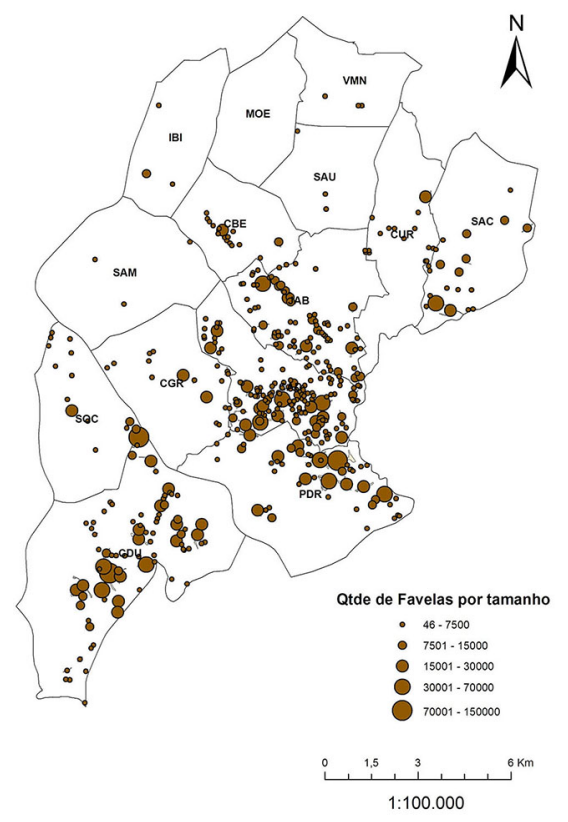

Fonte: Base Cartográfica Digital das Favelas do Município de São Paulo (PMSP/CEM, 2003).

Figura 3 - Índice de Desenvolvimento Humano (IDH) no setor, município de São Paulo, SP, Brasil no ano de 2000

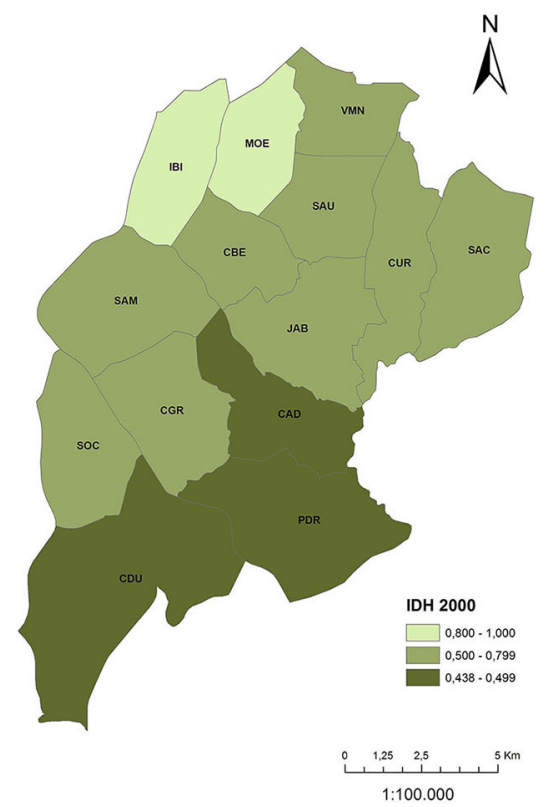

Fonte: www2.uol.com.br/aprendiz/n_noticias/.../id150802.doc 
Figura 4 - Perfil socioambiental no setor, município de São Paulo, SP, Brasil

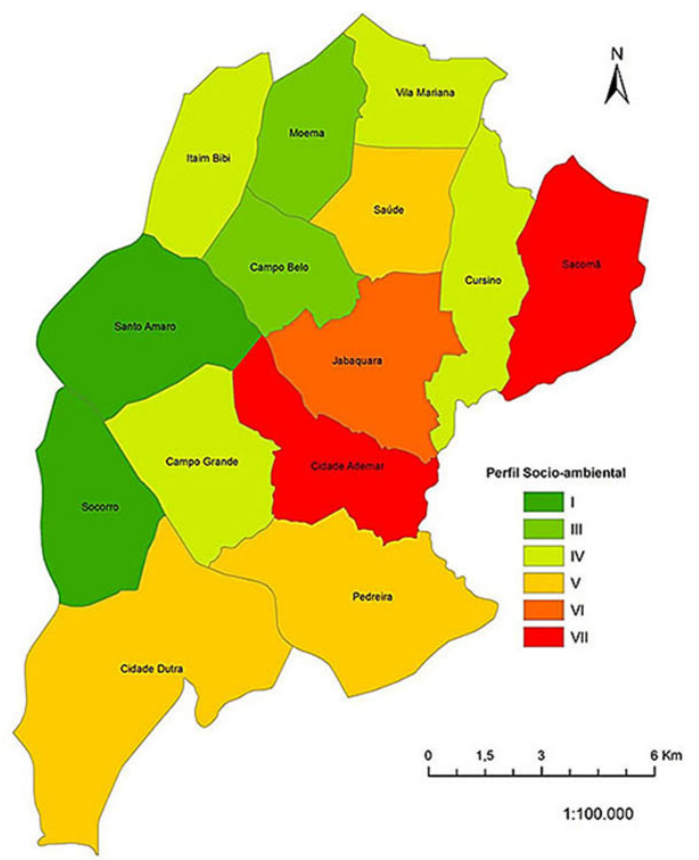

Fonte: SVMA (2004).

Figura 5 - Proporção da população (\%) estimada de crianças menores de 5 anos, por distrito, no setor, município de São Paulo, SP, no ano de 2005

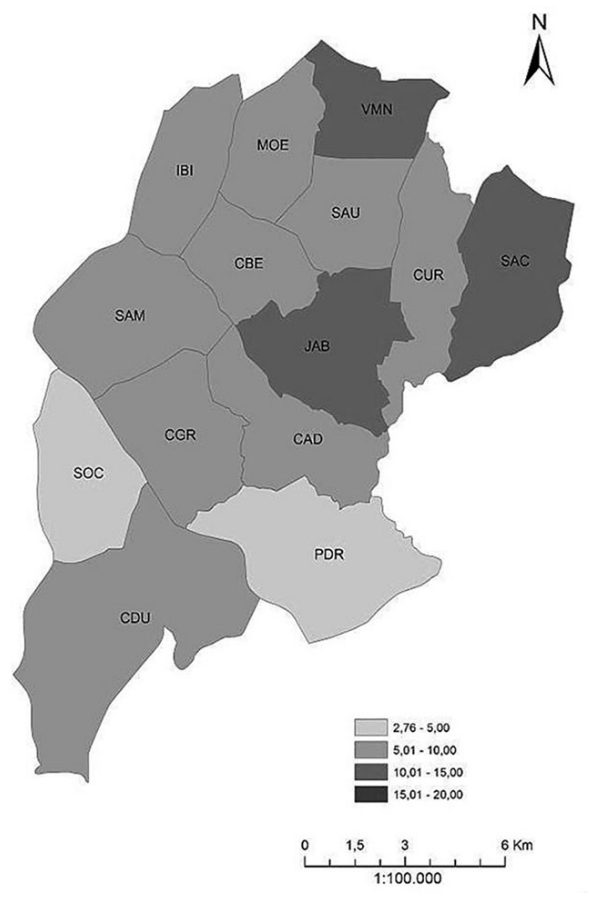

Fonte: Fundação Seade (2009). 
Figura 6 - Proporção da população (\%) estimada de idosos, por distrito, no setor, município de São Paulo, SP, no ano de 2005

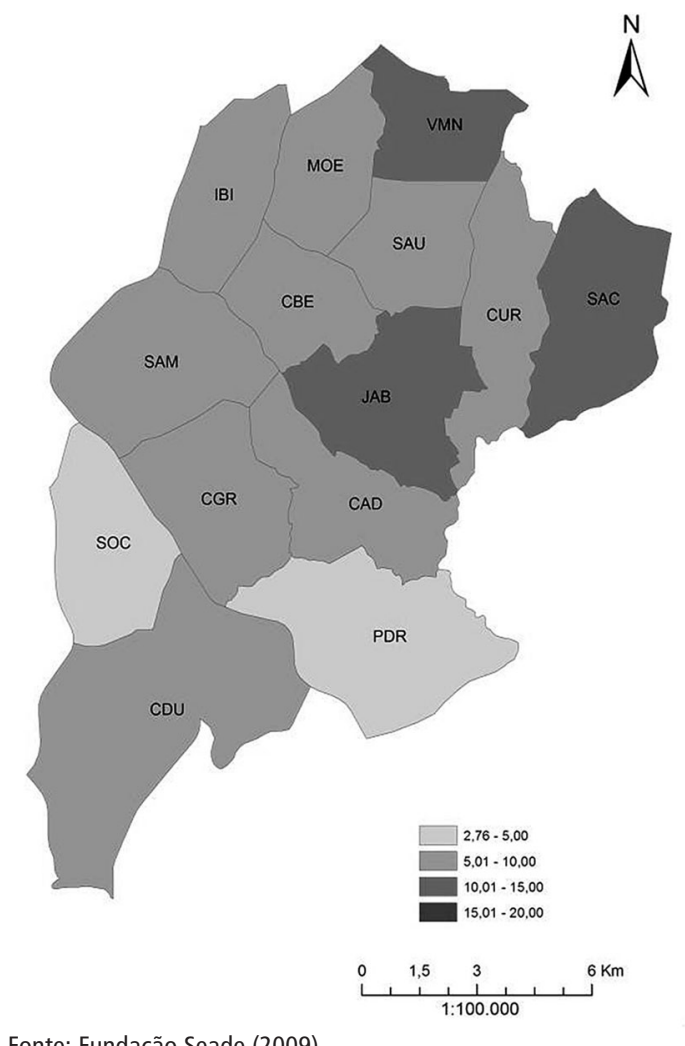

Fonte: Fundação Seade (2009).

encontrar áreas residenciais nobres, como os bairros arborizados, com residências baixas ou verticalizados; áreas industriais mais antigas, como a região de Santo Amaro, Interlagos; áreas com forte adensamento de construção e verticalização, como o eixo da Av. Luiz Carlos Berrini.

Há, igualmente, amplos espaços verdes preservados - como a região do Parque das
Fontes do Ipiranga, onde se localizam o Parque Zoológico e o Jardim Botânico - e as áreas de ocupação mais precárias - como as favelas e os bairros de ocupação mais recente nas bordas das represas Guarapiranga e Billings.

Esse mosaico confere, aos distritos, perfil heterogêneo, com relação a todas as variáveis: população, ambiente, grau de urbanização, entre outros (Figuras 7 e 8). 
Figura 7 - Vista parcial do distrito de Moema, melhor condição socioambiental, abril de 2010

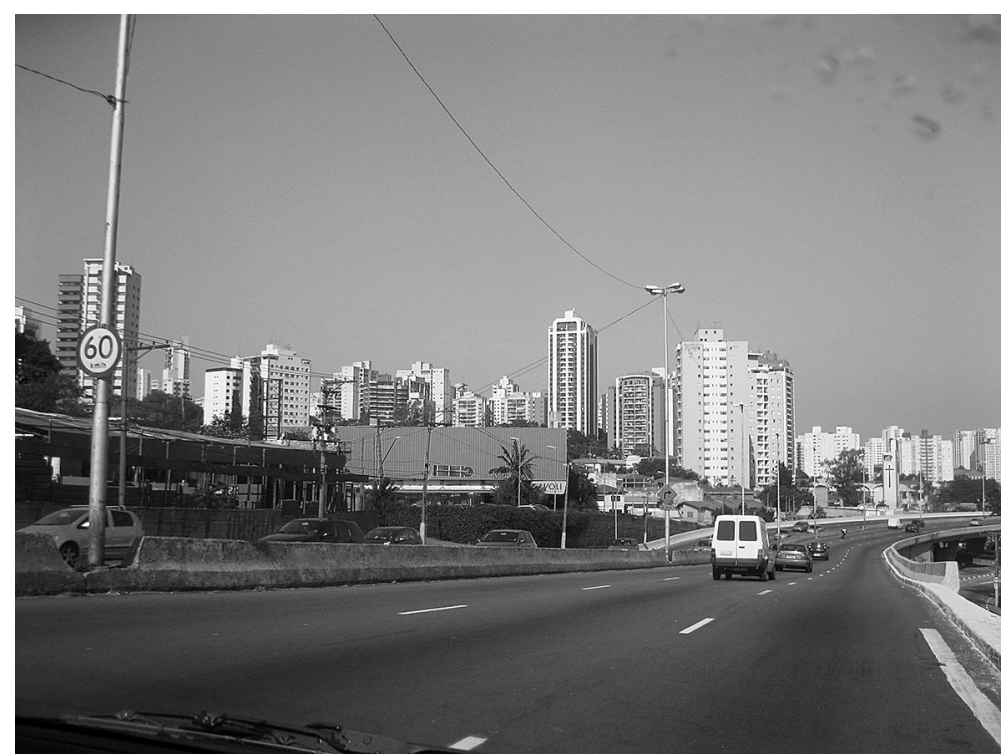

Fonte: Silva, E. N.

Figura 8 - Vista parcial do distrito de Cidade Ademar, pior condição socioambiental, abril de 2010

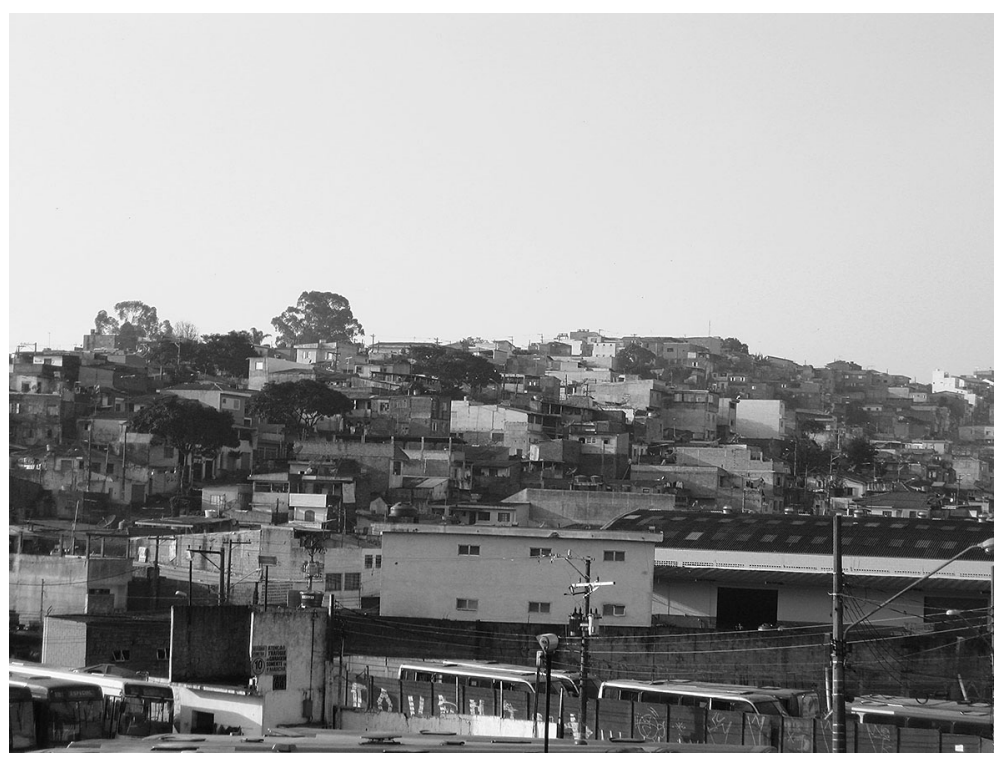

Fonte: Silva, E. N. 


\section{Resultados e discussão}

Os mapas (Figuras 9, 10 e 11) apresentam a espacialização das internações hospitalares por doenças respiratórias, em crianças e idosos, e por doenças circulatórias em idosos.

Os distritos Sacomã, Jabaquara, Cidade Ademar, Pedreira e Cidade Dutra foram aqueles que apresentaram as mais altas taxas de internação por doenças circulatórias, no período de 2003 a 2006. As maiores taxas restringiram-se aos distritos do Jabaquara, Cidade Ademar e Pedreira. Esses distritos contêm maior concentração de favelas da área estudada, menores índices de IDH, correspondendo à metade do indicador em relação aos distritos melhores posicionados e aos piores indicadores socioambientais.

Os distritos com as menores taxas de internação - entre 74,6 a 150,0 internações por 10.000 habitantes - foram Ibirapuera, Moema, Saúde, Campo Belo, em todo o período estudado. 0 distrito Cursino apresentou taxas baixas, no ano de 2003 e 2004, e o distrito Socorro, no ano de 2003. Os distritos restantes apresentaram taxas intermediárias de internação, em todo o período (Figura 11).

Desde 1940, estudos têm sido realizados, em São Paulo, a fim de compreender as causas das cardiopatias. A hipertensão foi apontada como principal causa das doenças do coração à época (Chiaverini, 1951). Estudo, recente,

Figura 9 - Internações por doenças do aparelho respiratório em crianças menores de 5 anos, no setor Sul-Sudeste, município de São Paulo, 2003 a 2007
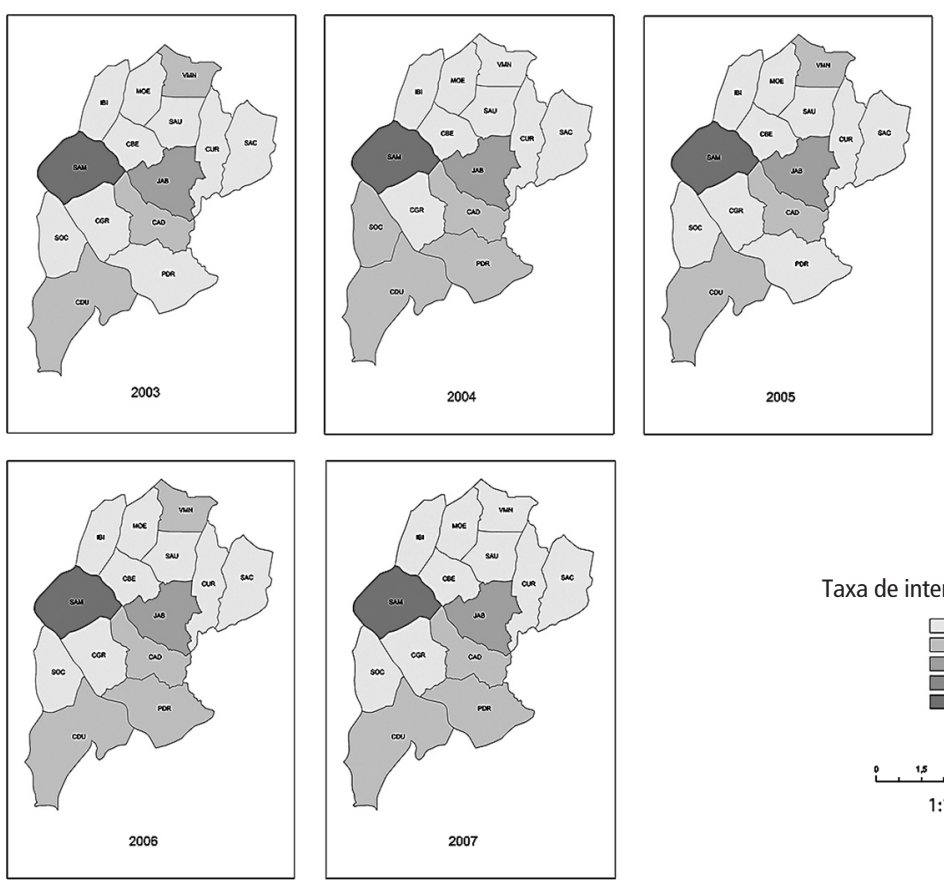

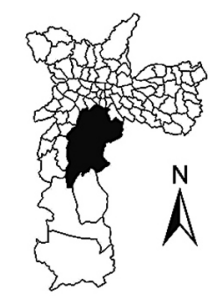

Taxa de internação por 10.000 hab

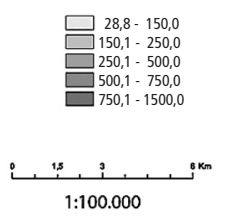


Figura 10 - Internações por doenças do aparelho respiratório em pessoas de 60 e mais anos, no setor Sul-Sudeste, município de São Paulo, 2003 a 2007
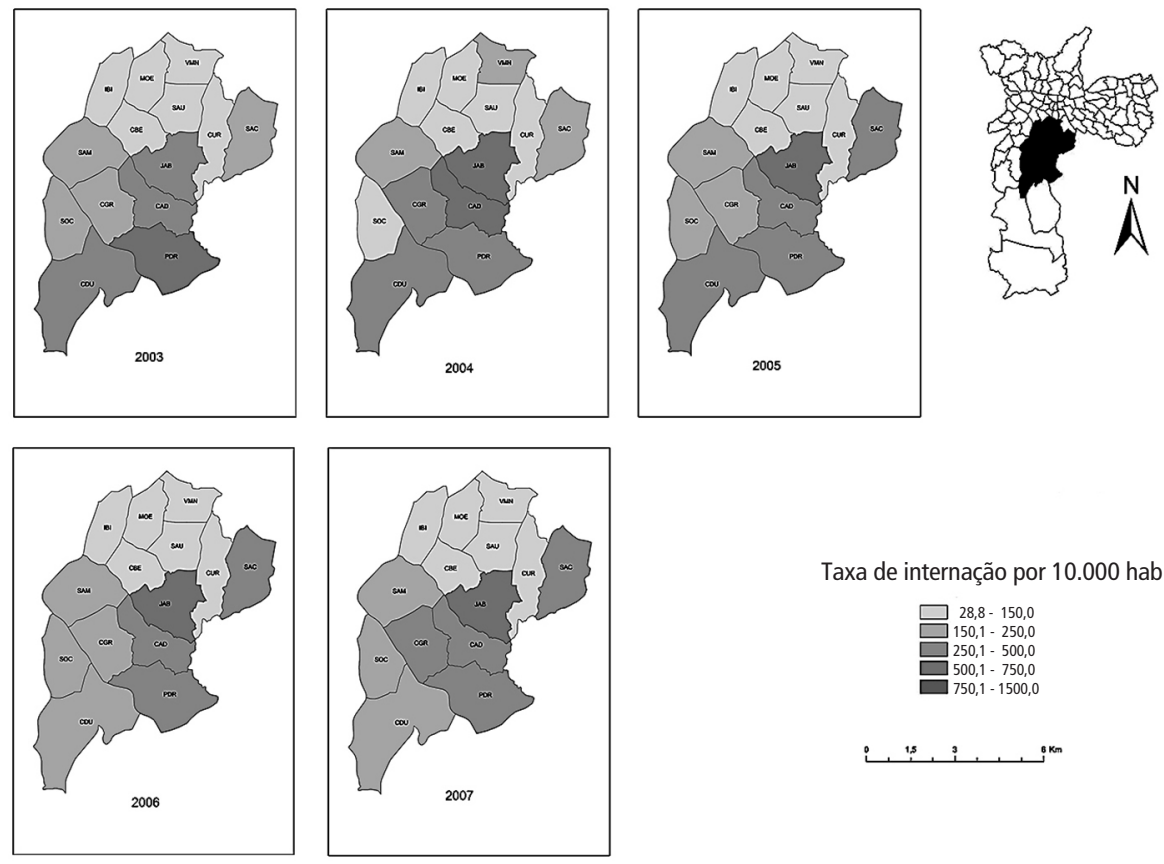

Taxa de internação por 10.000 hab

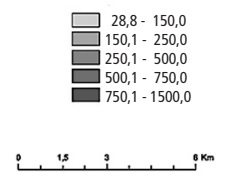

Figura 11 - Internações por doenças do aparelho circulatório em pessoas de 60 e mais anos, no setor Sul-Sudeste, município de São Paulo, 2003 a 2007
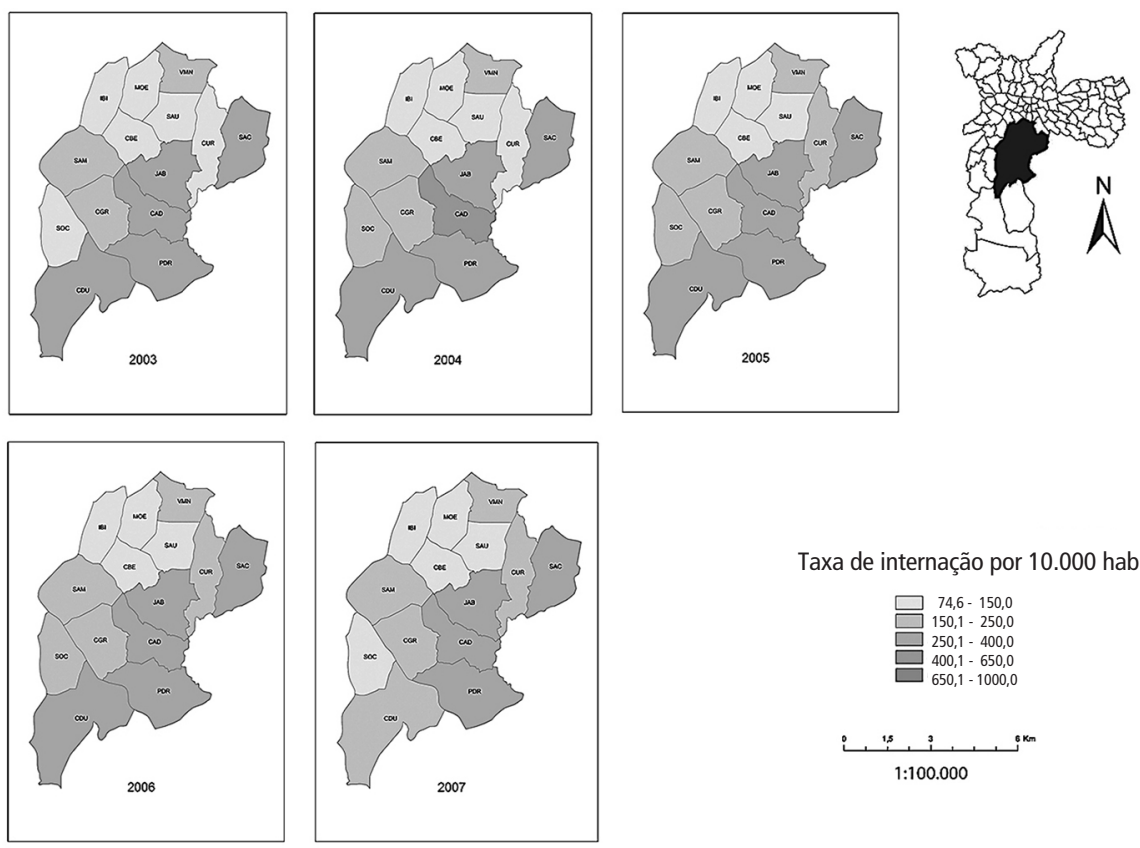

Taxa de internação por 10.000 hab

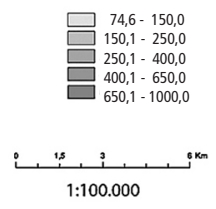


apontou o aumento das doenças hipertensivas na população brasileira, no período de 2000 a 2010 (Luiz et al., 2015).

Atualmente, sabe-se que a etiologia da doença é múltipla e extremamente comple$\mathrm{xa}$, e alguns de seus fatores de risco clássicos são: o hábito de fumar, dieta rica em gorduras e carboidratos refinados, sedentarismo e consequentemente a hipertensão, a diabetes e a obesidade (ibid.).

Estudo realizado pela Inteheart (Rosengren et al., 2004), em 52 países, mostrou que os fatores psicossociais estressantes, como: estresse no trabalho, tristeza, depressão, tensão, ansiedade devido a fatores externos, estão relacionados ao maior risco de ocorrência do infarto do miocárdio.

Estudos recentes mostram que a taxa de mortalidade por doenças do aparelho circulatório, mais especificamente, as cardiovasculares, vêm decrescendo no País (Luiz et al., 2015; Curioni et al., 2009; Ripsa, 2008) e na cidade de São Paulo (Lotufo, 2004). Curioni et al. (2009) mostraram que houve queda, em média de 3,9\% ao ano, nas taxas de mortalidade por doenças cardiovasculares em todas as faixas etárias, entre 1980 e 2003. Dados do estudo de Luiz et al. (2015) apontam redução de 10\% nas mortes por doenças circulatórias, no País, no período de 2000 a 2010.

[...] nossos dados mostram que a taxa de mortalidade por doenças do aparelho circulatório vem caindo. Com 226 óbitos por 100.000 homens e 169 por 100.000 mulheres no ano de 2010, as informações aqui apresentadas são semelhantes às estimadas pela Organização Mundial de Saúde para maioria dos países da Europa Ocidental e Austrália, cujas taxas se situam em torno de 240 por 100.000 homens e 180 por 100.000 mulheres. (Luiz et al., 2015, p. 345)

Os estudos mencionados apontam que as regiões mais ricas do País - Sul e Sudeste - apresentam um declínio mais acentuado na mortalidade por doenças cardiovasculares desde 1990. Os pesquisadores consideram que, nessas regiões, maior acesso à tecnologia médica (medicamentos, diagnósticos, etc.), programas de atendimento, além das campanhas de conscientização relacionadas ao hábito de fumar, podem estar relacionados ao declínio mais acentuado.

Além disso, a distribuição espacial de internações por doenças circulatórias, apresentada neste estudo, aponta um padrão socioespacial, ou seja, maiores taxas estão relacionadas aos distritos com pior perfil socioambiental e baixo IDH, e taxas menores estão associadas aos distritos com melhor perfil socioambiental e alto IDH. Barata (2012) argumenta que a produção e a distribuição das doenças e eventos relacionados à saúde são fenômenos complexos e nem sempre as explicações de sua ocorrência revelam-se a partir dos comportamentos individuais, da estratificação de classe, por exemplo. A autora aponta que a utilização da categoria espaço geográfico como indicador das condições de vida constitui uma vantagem para compreender a complexidade da relação saúde-doença. Na presente pesquisa, a distribuição espacial das internações hospitalares aponta para a desigualdade em saúde, indicando que os processos da produção do espaço estão relacionados às condições de saúde da população.

Esse mesmo padrão foi observado em Silva e Ribeiro (2009) para os dados de 
mortalidade por doenças circulatórias, no município de São Paulo, no mesmo período. 0 mapeamento indicou diferença socioespacial nos padrões de adoecimento e morte. Os dados de mortalidade representam universo mais amplo, ou seja, todos aqueles residentes da área que morreram pela doença no período. Similarmente, o mapeamento mostrou que a taxa de mortalidade por doenças do aparelho circulatório é mais elevada nos distritos com piores indicadores sociais, econômicos e ambientais. Os distritos de Santo Amaro e Cidade Ademar apresentam taxas elevadas de mortalidade por doenças circulatórias no período de 2003 a 2007 (Silva e Ribeiro, 2009).

A distribuição das internações por doenças do aparelho respiratório em pessoas de 60 anos ou mais é apresentada nos mapas da Figura 10. Nota-se que as maiores taxas de internação não obedecem a um padrão homogêneo, no período de 2003 a 2007. 0 distrito do Jabaquara apresentou as maiores taxas, no período de 2004 a 2007; o distrito de Pedreira, em 2003, e o distrito Cidade Ademar em 2004. Os distritos do Ibirapuera, Moema, Campo Belo, Saúde e Cursino apresentaram as menores taxas de internação durante todo o período estudado. Esses distritos apresentam as melhores condições de IDH e a menor concentração no número de favelas no interior do distrito.

Nesse caso, também a distribuição espacial aponta a desigualdade em saúde, ou seja, maiores taxas relacionadas aos distritos com pior perfil socioambiental e baixo IDH e menores taxas relacionadas aos distritos com melhor perfil socioambiental e alto IDH. Considera-se, porém, que essa distribuição pode estar relacionada com o aumento mais recente da população idosa em distritos mais pobres ou com o maior poder aquisitivo da população residente em distritos de melhor condição socioeconômica, permitindo o uso de sistema ou de planos privados de saúde. Considerando a última hipótese, ressalta-se que dados sobre internações hospitalares realizadas na rede privada não constam neste estudo, o que é uma limitação dos dados.

A Figura 9 apresenta os mapas de internação por doenças respiratórias, em crianças menores de 5 anos. 0 distrito de Santo Amaro apresentou as maiores taxas de internação, por todo o período estudado - entre 750,1 e 1500,0 internações por 10.000 habitantes -; seguido do distrito do Jabaquara, que apresentou taxas entre 500,1 a 750 internações por 10.000 habitantes. Esses distritos não apresentam os piores indicadores de IDH e socioambiental, e somente o distrito do Jabaquara apresenta grande concentração de áreas de favela em seu território. Os distritos do Ibirapuera, Moema, Saúde, Campo Belo, Cursino, Campo Grande e Sacomã apresentaram taxas baixas por todo período estudado - 2003 a 2007 - entre 28,8 e 150,0 internações por 10.000 habitantes.

A espacialização dos dados apontou que não há um padrão socioespacial definido das internações de crianças. Em outras palavras, taxas elevadas podem ocorrer tanto em distritos com baixo IDH e pior perfil socioambiental, como em distritos de médio e alto IDH e de melhor perfil socioambiental. Nesse sentido, ressaltam-se as taxas elevadas ocorridas no distrito de Vila Mariana de alto padrão em 2003, 2005 e 2006.

No município de São Paulo, dois inquéritos realizados entre 1984/1985 e 1995/1996, que avaliaram a evolução das doenças respiratórias em crianças menores de 5 anos, na 
cidade, observaram aumentos substanciais e generalizados para doença respiratória alta e para a doença respiratória baixa sem chiado. $\mathrm{A}$ pesquisa mostrou um acréscimo de $71 \%$ para o conjunto dos casos, de $66 \%$ para os casos de doença alta ou de doença baixa sem chiado, e $250 \%$ para os casos de doença baixa com chiado, cujo aumento foi muito grande para as crianças pertencentes ao terço mais pobre da população (Benicio et al., 2000; Barata et al., 1996; Monteiro e Benicio, 1987).

Estudo apontou a densidade de ocupação no domicílio como um dos fatores do aumento das doenças respiratórias em crianças. Contudo, a evolução das condições socioeconômicas e as condições de salubridade do meio ambiente, contraditoriamente, não foram capazes de explicar a duplicação da doença respiratória baixa na população das crianças menores de 5 anos, da cidade de São Paulo (Benicio et al., 2000).

Estudo, que analisou a associação entre morbidade, variáveis climáticas e índice de conforto, apontou que extremos de frio e de calor representaram maior risco para internações de idosos, e os distritos com piores condições sociais e ambientais (Silva e Ribeiro, 2012). No entanto, a associação dessas variáveis atmosféricas com as internações de crianças com doenças respiratórias e menores de 5 anos residentes não apresentou diferença em relação às condições socioambientais (Silva e Ribeiro, 2013).

Os resultados relacionados à internação de crianças por doenças respiratórias, nos distritos apresentados, ressaltam a complexidade do fenômeno saúde-doença, pois esta não apresentou uma relação causal direta entre internações e piores condições socioambientais.
Outros fatores, como a poluição atmosférica, a presença de ácaros no domicílio e a frequência em creches, tendem a aumentar com o progresso econômico das sociedades e dos indivíduos, evidenciando a não simplicidade dessa relação (Benicio et al., 2000).

\section{Conclusões}

Este trabalho mostrou a desigual distribuição espacial das internações hospitalares de idosos e crianças, em hospital público, em 14 distritos do município de São Paulo. 0 georreferenciamento, por endereço de residência do paciente, foi possível devido à disponibilidade dos dados de internação diários, com Código de Endereçamento Postal (CEP) de residência, na rede mundial de computadores. Esses dados permitem localizar, no espaço, um grande volume de informações sobre determinados desfechos de saúde - nesse caso internações hospitalares por doenças do aparelho respiratório e circulatório - e contribuem para a identificação das desigualdades e iniquidades.

A disponibilidade de dados e a facilidade de acesso às informações permitem estudar a distribuição e a magnitude das doenças crônicas, em diversas escalas espaciais, bem como associá-las a fatores de risco, a fim de melhor entender seus determinantes e poder planejar e direcionar as ações de promoção de saúde e de busca da equidade.

0 estudo demostrou que a distribuição espacial das taxas de internação hospitalar não apresenta padrão homogêneo entre os distritos. No entanto, deve-se ressaltar que, no município de São Paulo, 50\% dos residentes são usuários exclusivos do SUS e também que 
não estão distribuídos igualmente no espaço. 0 maior número de usuários do SUS está concentrado em distritos de pior condição socioeconômica, refletindo as desigualdades sociais na cidade de São Paulo. Além disso, o SUS baseia-se nos princípios de universalidade, equidade e integralidade, visando à superação das iniquidades, que são as desigualdades injustas e evitáveis, em saúde. As diferenças em "saúde injustas são aquelas que estão associadas a características sociais que sistematicamente colocam alguns grupos em desvantagem com relação à oportunidade de ser e de se manter sadio" (Barata, 2012). As desigualdades evitáveis são aquelas para as quais existem tecnologias médicas e de saneamento, que poderiam evitar 0 adoecimento de um grupo populacional, mas que não estão disponíveis àquele grupo, por conta de sua posição social, renda ou local de moradia.

No entanto, o padrão de espacialização intraurbano da morbidade, no setor da cidade estudado, aponta para diferenças importantes e que merecem aprofundamento na investigação e atenção de gestores de saúde pública para seu enfrentamento.

\section{Helena Ribeiro}

Universidade de São Paulo, Faculdade de Saúde Pública, Departamento de Saúde Ambiental. São Paulo, SP/Brasil. lena@usp.br

\section{Edelci Nunes Silva}

Universidade Federal de São Carlos, Centro de Ciências Humanas e Biológicas, Departamento de Geografia, Turismo e Humanidades. Sorocaba, SP/Brasil.

enunes@ufscar.br

\section{Referências}

AITH, F. e SCALCO, N. (2015). Direito à saúde de pessoas em condições de vulnerabilidade em centros urbanos. Revista USP, n. 107, pp. 41-54.

ALMEIDA, E. (2001). "Refuncionalização da metrópole no período técnico-científico-informacional e os novos serviços". In: SANTOS, M. e SILVEIRA, M. L. O Brasil. Território e sociedade no início do século XXI. Rio de Janeiro/São Paulo, Record.

BARATA, R. C. B. et al. (1996). Gastroenterites e infecções respiratórias agudas em crianças menores de 5 anos em área da região Sudeste do Brasil, 1986-1987. I- Infecções respiratórias agudas. Revista de Saúde Pública, v. 30, n. 6, pp. 553-563. 
BARATA, R. B. (2012). Como e por que as desigualdades sociais fazem mal à saúde. Rio de Janeiro, Editora Fiocruz.

BENICIO, M. H. D. A et al. (2000). Tendência secular da doença respiratória na infância na cidade de São Paulo (1984-1996). Revista de Saúde Pública. São Paulo, v. 34, n. 6, pp. 91-101. Suplemento.

CEInfo (2010). Coordenação de Epidemiologia e Informação. Estimativa da População Exclusivamente Usuária SUS No Município de São Paulo. Boletim Eletrônico CEInfo, ano 1, Boletim 1. Disponível em: http://www.prefeitura.sp.gov.br/cidade/secretarias/upload/saude/arquivos/ boletimeletronico/n01popsus.pdf. Acesso em: 2 mar 2016.

CHIAVERINI, R. (1951). Tipos etiológicos de cardiopatia em um núcleo ferroviário de São Paulo. Arquivos Brasileiros de Cardiologia, n. 4, pp. 403-412.

CURIONI, C. et al. (2009). The decline in mortality from circulatory diseases in Brazil. Rev. Panam Salud Publica, v. 25, n. 1.

DATASUS (2016). Sistema de Informações Hospitalares do Sistema Único de Saúde (SIH/SUS). Disponível em: http://www2.datasus.gov.br/SIHD/. Acesso em: 2 mar 2016.

IBGE - INSTITUTO BRASILEIRO DE GEOGRAFIA E ESTATÍTICA (2007). Contagem da População. Disponível em: ftp://ftp.ibge.gov.br/Contagem_da_Populacao_2007. Acesso em: 30 jan 2016.

(2015). Estimativa da População. Disponível em: http://www.cidades.ibge.gov.br/xtras/perfil. php?lang=\&codmun=355030. Acesso em: 30 jan 2016.

JARA, J. J.; HIDALGO, M. T. e HANSEN, R. S. (2010). "La ciudad desde los determinantes de la salud". In: GALVÃO, L. A. C.; FINKELMAN, J. e HENAO, S. (2010). Determinantes ambientales y sociales de la salud. Washington, D.C., Organización Panamericana de la Salud.

LOTUFO, P. A. (2004). Por que não vivemos uma epidemia de doenças crônicas: o exemplo das doenças cardiovasculares? Ciência \& Saúde Coletiva, v. 9, n. 4, pp. 841-850.

LOYOLA, E.; CASTILLO-SALGADO, C.; NÁJERA-AGUILAR, P. et al. (2002). Los sistemas de información geográfica como herramienta para monitorear las desigualdades de salud. Revista Panamericana Salud Pública, v. 12, n. 6, pp. 415-428.

LUIZ, O. C.; LOUZADA, M. L. da C. e FONSECA, L. A. M. (2015). "Doenças do aparelho circulatório: a redução da mortalidade (2000-2010)". In: MONTEIRO, C. A. e LEVY, R. B. (orgs.). Velhos e novos males da saúde no Brasil: de Geisel a Dilma. São Paulo, Hucitec/Nupens/USP.

MONTEIRO, C. A. e BENICIO, M. H. A. (1987). Estudo das condições de saúde das crianças no município de São Paulo, SP (Brasil) 1984/1985. VI. Doença Respiratória. Revista Saúde Pública. São Paulo, v. 21, n. 5 , pp. $380-386$.

OPAS - ORGANIZACIÓN PANAMERICANA DE LA SALUD (2011). 51ํo Consejo Directivo. Estrategia y Plan de acción sobre la Salud Urbana. Washington D.C., EUA.

PERES, M. F. T. e RUOTTI, C. (2015). Violência urbana e saúde. Revista USP, n. 107, pp. 65-78.

RIBEIRO, H. (2006). "Patologias do ambiente urbano: desafios para a Geografia da Saúde”. In: SILVEIRA, M. L. et al. (orgs.). Questões territoriais na América Latina. CLACSO Livros/Depto. de Geografia da Universidade de São Paulo, pp. 277-293.

RIBEIRO, H. e VARGAS, H. (2015). Urbanização, globalização e saúde. Revista USP, n. 107, pp. 13-26. 
RIPSA - REDE INTERAGENCIAL DE INFORMAÇÃO PARA SAÚDE (2008). Indicadores Básicos para a Saúde no Brasil: Conceitos e Aplicações. Disponível em: http://www.ripsa.org.br/php/level.php?lang=pt \&component=68\&item=20. Acesso em: 6 jan 2010.

ROSENGREN, A. et al. (2004). Association of psychosocial risk factors with risk of acute myocardial infarction in 11119 cases and 13648 controls from 52 countries (the INTERHEART study): casecontrol study. The Lancet, v. 364, pp. 953-962.

SANTOS, M. (1996). A urbanização brasileira. São Paulo, Hucitec.

(2003). Saúde e ambiente no processo de desenvolvimento. Ciência \& Saúde Coletiva, v. 8, n. 1, pp. 309-314.

SANTOS, M. e SILVEIRA, M. L. (2001). O Brasil. Território e sociedade no início do século XXI. Rio de Janeiro/São Paulo, Record.

SEADE (2016). Informações dos Municípios Paulistas. São Paulo. Disponível em: http://www.imp. seade.gov.br/frontend/\#/perfil. Acesso em: 11 maio 2016.

SEPE, P. M. e TOKIYA, H. (coords.) (2004). Atlas Ambiental do Município de São Paulo - O Verde, o Território, o Ser Humano: diagnóstico e bases para a definição de políticas públicas para as áreas verdes no município de São Paulo. São Paulo, SVMA - SECRETARIA MUNICIPAL DO VERDE E MEIO AMBIENTE.

SILVA, E. N. e RIBEIRO, H. (2009). Perfil Intraurbano da morbi-mortalidade por doenças cardiovasculares no grupo etário acima de 60 anos no município de São Paulo. In: 11 CONGRESSO PAULISTA DE SAÚDE PÚBLICA: SAÚDE E CRISE(S) FRONTEIRAS E CAMINHOS. São José dos Campos.

(2012). Impact of urban atmospheric environment on hospital admissions in the elderly. Revista de Saúde Pública, v. 46, n. 4, pp. 694-701.

(2013). Medio ambiente atmosférico urbano e ingresos hospitalarios de niños en la ciudad de Sao Paulo, Brasil. Revista de Salud Ambiental, v. 13, pp. 30-36.

SOUZA-SANTOS, B. (2002). “Os processos da globalização”. In: SOUZA-SANTOS, B. A globalização e as ciências sociais. São Paulo, Cortez.

TASCHNER, S. P. (2001). “Um olhar sobre o urbano: políticas públicas em São Paulo”. In: SORRENTINO, M. Ambientalismo e participação na contemporaneidade. São Paulo, Educ/Fapesp.

Texto recebido em 4/fev/2016 Texto aprovado em 25/abr/2016 
